\section{Traveling Wave Tube-Based LINC Transmitters}

\author{
A. Choffrut, B. D. Van Veen, and J. H. Booske
}

\begin{abstract}
Traveling wave tubes (TWTs) have unparalleled power efficiency in the microwave frequency regime and above. Linear amplification with nonlinear components (LINC) is a transmission architecture capable of achieving both power and spectral efficiency. By using amplifiers operated near saturation, LINC has drawn great attention in the solid state community, but has not been explored in the context of high power TWT applications. We demonstrate that in the presence of realistic imperfections the LINC TWT transmitter achieves $-45 \mathrm{dBc}$ or better spectral regrowth while operating under saturated, maximum efficiency conditions. In our simulations, the amplifier model is based on actual TWT measurements.
\end{abstract}

Index Terms-Amplifier distortion, LINC, nonlinear distortion, nonlinearities, traveling wave tubes.

\section{INTRODUCTION}

A novel transmission scheme known as linear amplification using nonlinear components (LINC), is introduced in [2]. Under ideal conditions, a LINC transmitter performs perfect linear amplification with any type of amplifier. Furthermore in a LINC transmitter the amplifiers can be operated, in theory, at saturation without producing unwanted spectral regrowth. As shown in Fig. 1, the LINC transmitter consists of a signal component separator (SCS) which divides the bandlimited source signal into two broadband constant amplitude, or phase-modulated, signals. The inband components of these two signals add up to the source signal and the out-of-band components cancel because they are equal in magnitude but $180^{\circ}$ out of phase. These components are separately upconverted to the carrier frequency using quadrature modulators and then amplified. Since the envelopes of both modulated signals are constant in magnitude, the amplifiers will not introduce nonlinear distortion, even at saturation. A power combiner sums the two amplified signals to reconstruct an amplified, undistorted version of the source signal.

LINC has been extensively studied in the context of solid-state devices and is arguably a very promising architecture in light of RF component quality and the impressive development of digital processors [6], [7]. The possibility of operating amplifiers at saturation in spite of their nonlinearities makes traveling wave tubes (TWTs) obvious candidates for LINC, owing to their attractive efficiency performance. Saturated TWT efficiencies of $65-75 \%$ are reported in the literature [1], [4]. The amplifiers of a LINC transmitter are continuously operated at an approximately constant reference drive level. Thus, the TWT amplifiers may be operated at a very low back-off, where efficiency is close to its peak.

The misalignments present in a real modulator corrupt the envelope of the signals during upconversion [5], [7] and consequently the amplifiers are not operated at a fixed point in a realistic LINC transmitter. The nonlinear characteristics of the amplifiers are then apparent and signal energy appears outside the source signal bandwidth at the output of the transmitter. A second problem with realistic LINC transmitters is

Manuscript received October 17, 2002; revised December 23, 2002. This work was supported in part by AFOSR, and by DUSD (S\&T) under the Innovative Microwave Vacuum Electronics Miltidisciplinary University Research Initiative (MURI) program, managed by AFOSR. The review of this brief was arranged by Editor D. Goebel.

The authors are with the Electrical and Computer Engineering Department, University of Wisconsin, Madison, WI USA 53706 (e-mail: choffrut@cae.wisc.edu; vanveen@engr.wisc.edu; booske@engr.wisc.edu).

Digital Object Identifier 10.1109/TED.2003.813459

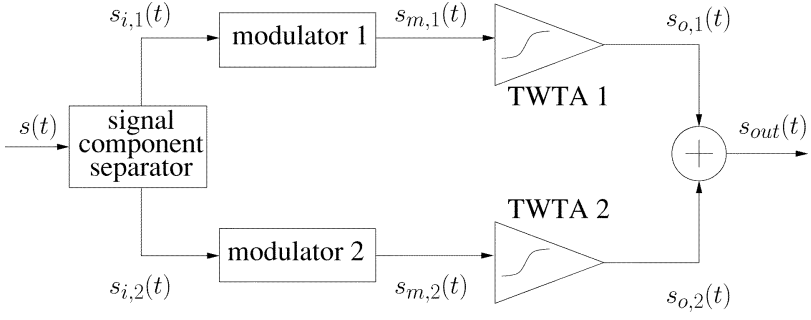

Fig. 1. LINC transmitter (signals are denoted by their envelopes) SCS generates two phase-modulated base-band signals, which are upconverted in two modulators, and then amplified using two TWT amplifiers. The amplified signals are summed in a power combiner. The output is a linearly amplified modulated version of the input signal.

mismatch between the two amplifier characteristics. As with modulator misalignment, mismatch in the amplifiers results in imperfect cancellation of the out-of-band signal components. The impact of imperfections in the modulators on solid-state amplifier based LINC schemes is reported in [7]. The purpose of this letter is to demonstrate that the LINC transmitter has significant potential for TWT applications by evaluating both the impact of realistic modulator misalignment and TWT amplifier mismatch.

The notation and mathematical models are defined in Section II. In Section III, the procedure employed to simulate LINC transmitter performance is described. Section IV presents the simulated output spectra of a LINC transmitter in three scenarios: In the first the amplifiers are identical, but the modulators are impaired; in the second the amplifiers are mismatched and the modulators ideal. Additional simulations illustrate the impact of the relative values between misalignment parameters in both modulators. The third scenario combines amplifier mismatch and modulator imperfections. For comparison a single-modulator, single-amplifier transmitter, referred to as the "no LINC transmitter," is also simulated.

\section{NOTATIONS AND MODELS}

This section introduces standard notation and models from [3] and [7] that are used later in the description of LINC.

\section{A. Complex Baseband Representation of Real Passband Signals}

A real, passband signal $z(t)$ at carrier frequency $f_{c}$ is equivalently represented with its complex, baseband envelope $s(t)=x(t)+j y(t)$. The $I$ - and $Q$-components $x(t)$ and $y(t)$ modulate the carrier [3]

$$
z(t)=x(t) \cos \left(2 \pi f_{c} t\right)-y(t) \sin \left(2 \pi f_{c} t\right) .
$$

\section{B. Amplifier Transfer Curves}

The AM/AM and AM/PM transfer curves give the relationship between envelopes of the input and the output of an amplifier in terms of the change in amplitude and phase introduced by the amplifier. Assume that the input to an amplifier with AM/AM characteristic $A(r)$ and $\mathrm{AM} / \mathrm{PM}$ characteristic $\Delta \phi(r)$ is a single tone:

$$
z(t)=r \cos \left(2 \pi f_{c} t+\theta\right)=x \cos \left(2 \pi f_{c} t\right)-y \sin \left(2 \pi f_{c} t\right)
$$

that is, the envelope is given by

$$
s(t)=r e^{j \theta}=x+j y
$$

where $r, \theta, x$, and $y$ are constant. The magnitude of the envelope is then $|s|=\left|r e^{j \theta}\right|=r=\sqrt{x^{2}+y^{2}}$. The output of the modulator is

$$
s_{o}(t)=A(r) \cdot e^{j(\theta+\Delta \phi(r))} \text {. }
$$


Hence, the gain of the amplifier is $G(r)=(A(r) / r) e^{j \Delta \phi(r)}$.

We assume a memoryless amplifier. These equations remain valid in the more general case of amplitude- and phase-modulated signals, provided the bandwidth of the signal is small compared to the carrier frequency. In other words, these equations are valid if $x$ and $y$, and $r$ and $\theta$ are time-varying as long as the rate of change is small compared to the carrier frequency.

\section{Model for the Modulators}

In a real modulator the $I$ - and $Q$-branches do not operate perfectly symmetrically on the components. The output of an impaired modulator with input as in (1) can be modeled [7] by

$$
\begin{aligned}
z_{m}(t)=\left(\left(1+g_{d}\right) x(t)\right. & \left.+p_{I}\right) \cos \left(2 \pi f_{c} t+\phi_{d}\right) \\
& -\left(\left(1-g_{d}\right) y(t)+p_{Q}\right) \sin \left(2 \pi f_{c} t-\phi_{d}\right)
\end{aligned}
$$

where $g_{d}$ is the differential gain and $\phi_{d}$ is the differential phase. The offsets $p_{I}$ and $p_{Q}$ will be integrated with other terms to give a single parameter, the $d c$ offset $p$. After algebraic manipulations, one finds the following expression for the complex envelope $s_{m}(t)$ of the modulated signal $z_{m}(t)[7]$

$$
s_{m}(t)=u s(t)+v \overline{s(t)}+p .
$$

$\overline{(\cdots)}$ denotes complex conjugation, and $p, u$, and $v$ are complex-valued

$$
\begin{aligned}
p= & {\left[p_{I} \cos \left(\phi_{d}\right)+p_{Q} \sin \left(\phi_{d}\right)\right] } \\
& +j\left[p_{I} \sin \left(\phi_{d}\right)+p_{Q} \cos \left(\phi_{d}\right)\right] \\
u= & \frac{1}{2}\left[\left(1+g_{d}\right) e^{j \phi_{d}}+\left(1-g_{d}\right) e^{-j \phi_{d}}\right] \\
v= & \frac{1}{2}\left[\left(1+g_{d}\right) e^{j \phi_{d}}-\left(1-g_{d}\right) e^{-j \phi_{d}}\right] .
\end{aligned}
$$

A flawless modulator has $g_{d}=0, \phi_{d}=0$, and $p=0$. In turn, $u=1$ and $v=0$ and $s_{m}(t)=s(t)$ : the envelope is preserved. We use $g_{d, 1}$, $\phi_{d, 1}$, and $p_{1}$ to denote imbalances within modulator 1 , and $g_{d, 2}, \phi_{d, 2}$ and $p_{2}$ to denote imbalances within modulator 2 .

\section{Ideal LINC}

Let $s(t)$ denote the band-limited source signal and $r$ its maximum amplitude. The SCS generates the two phase-modulated signals $s_{i, 1}$ and $s_{i, 2}$ as follows:

$$
\begin{aligned}
& s_{i, 1}(t)=s(t)+e(t) \\
& s_{i, 2}(t)=s(t)-e(t)
\end{aligned}
$$

where $e(t)=j s(t) \sqrt{\left(r^{2} /|s(t)|^{2}\right)-1}$. The upconverted phase-modulated signals have complex envelope

$$
s_{m, k}(t)=u_{k} s_{i, k}(t)+v_{k} \overline{s_{i, k}(t)}+p_{k}, \quad k=1,2 .
$$

Assuming the amplifiers have gain $G_{k}(\cdot)$, their outputs are

$$
s_{o, k}(t)=s_{m, k}(t) \cdot G_{k}\left(\left|s_{m, k}(t)\right|\right), \quad k=1,2 .
$$

Finally, the power combiner output is

$$
s_{\text {out }}(t)=s_{o, 1}(t)+s_{o, 2}(t) .
$$

If the modulators are perfect and the amplifiers are operated at a constant gain $G$, assumed equal in both amplifiers, then

$$
\begin{aligned}
& s_{o, 1}(t)=G s_{m, 1}(t)=G s_{i, 1}(t)=G(s(t)+e(t)) \\
& s_{o, 2}(t)=G s_{m, 2}(t)=G s_{i, 2}(t)=G(s(t)-e(t)) \\
& s_{\text {out }}(t)=2 G s(t) .
\end{aligned}
$$

Thus, in the ideal case, the output is a linearly amplified, modulated version of the input. In the presence of modulator misalignment and

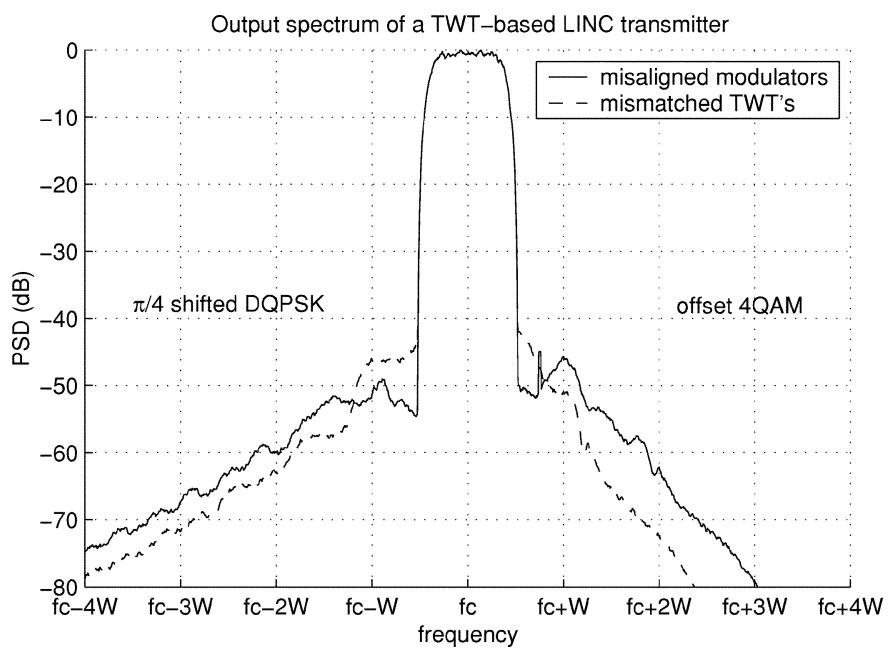

Fig. 2. Output spectra of a TWT-based LINC transmitters afflicted with amplifier mismatch only (dashed line), and with modulator misalignments only (solid line). Operation is at $6 \mathrm{~dB}$ gain compression point.

amplifier mismatch $e(t)$ present in $s_{o, 1}(t)$ and $s_{o, 2}(t)$, no longer cancels in $s_{\text {out }}(t)$. Since $e(t)$ is broadband, this results in spectral splatter outside the bandwidth of $s(t)$.

\section{Simulations}

Two typical modulation schemes are used: $\pi / 4$ shifted DQPSK as used in North American digital cellular systems and a variation thereof, offset 4QAM. These modulations are often said to be with constant envelope, because the points of their constellation are at an equal distance to the origin. However, when the pulse shape is not rectangular, as in any practical implementation, the actual instantaneous envelope of the signal varies over time.

The simulations are run in MATLAB. A square root raised cosine pulse shape is used with roll-off factor 0.35. The output spectra are estimated by averaging 128 spectra, each of which is obtained from a sequence of 128 random symbols, sampled at 32 samples per symbol. Then the averaged spectrum is further smoothed by averaging with the four adjacent points.

The gain for each amplifier is modeled by curve fitting measurements of actual TWT devices, provided by Boeing EDD. The measured complex gain is modeled as $G(r)=g_{1}+g_{3} \cdot r^{2}+g_{5} \cdot r^{4}$. The amplifiers are operated at the 6- $\mathrm{dB}$ gain compression point. Unless otherwise specified, we choose equal modulator errors in both branches, at about $150 \%$ of the lower bounds in [7]: $g_{d, 1}=g_{d, 2}=1 \% ; \phi_{d, 1}=\phi_{d, 2}=$ $0.5^{\circ} ; p_{1}=p_{2}=1.5 \%$.

\section{RESULTS}

Since the output spectra are symmetric, we show simulation results for $\pi / 4$ shifted DQPSK and offset 4QAM on the left- and right-hand sides (LHS or RHS) of Fig. 2, respectively. The spectra are plotted relative to the maximum level of the signal. The frequency range is $f_{c}-4 W$ to $f_{c}+4 W$, where $f_{c}$ is the carrier frequency and $W$ is the message bandwidth. In Fig. 2 LINC is simulated under two different conditions: in the first the amplifiers are identical but the modulators are impaired; in the second, the amplifiers are mismatched while the modulators are perfect.

The levels of spectral regrowth due to small impairments in the modulators (solid line) are on par with those caused by amplifier mismatch (dashed line). This suggests that it is just as important to find good modulators as it is to find amplifiers that are closely matched. With realistic modulators, the spectral splatter is kept under $-45 \mathrm{dBc}$ for offset 4QAM and under $-50 \mathrm{dBc}$ for $\pi / 4$ shifted DQPSK. Note that 


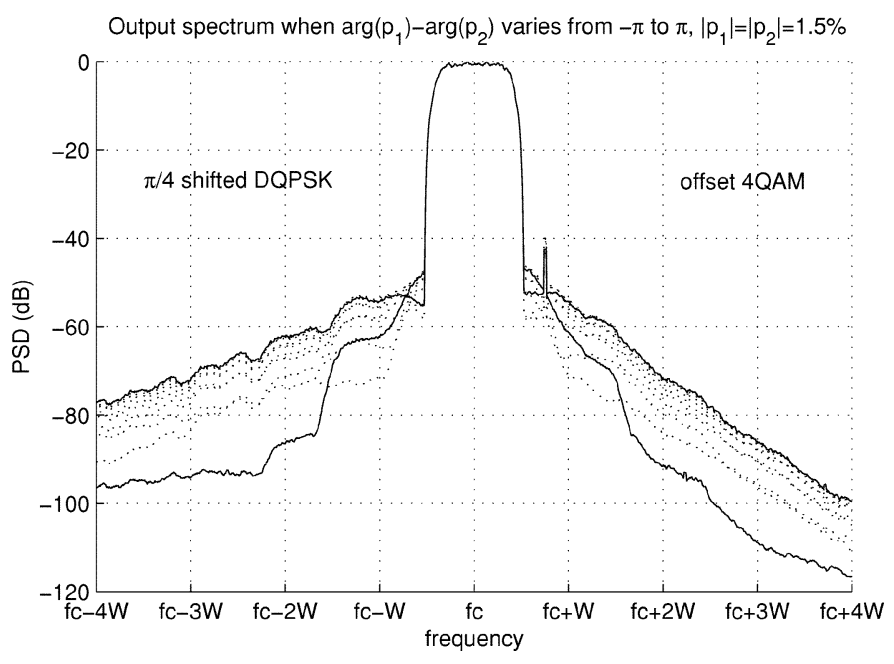

Fig. 3. Output power spectrum as a function of the relative angle between $\boldsymbol{p}_{1}$ and $\boldsymbol{p}_{2}:\left|\boldsymbol{p}_{1}\right|$ and $\left|\boldsymbol{p}_{2}\right|$ are kept at $1.5 \%$ while $\arg \left(\boldsymbol{p}_{1}\right)-\arg \left(\boldsymbol{p}_{2}\right)$ varies from $-\pi$ to $\pi$. Operation is at $6 \mathrm{~dB}$ gain compression point.

the residual spectrum decays faster for offset 4QAM. Also, residual spectrum due to amplifier mismatch is at slightly higher levels than that due to modulator misalignments at frequencies adjacent to the message bandwidth. Otherwise, when $\left|f-f_{c}\right|>1.5 \mathrm{~W}$ for $\pi / 4$ shifted DQPSK and $\left|f-f_{c}\right|>W$ for offset 4QAM, residual spectrum due to modulator misalignments is at higher levels.

We now discuss the individual roles of the error parameters $p, g_{d}$ and $\phi_{d}$. As in [7] with class C amplifiers, simulations not shown in this paper indicate that in the case of TWT amplifiers the contributions of the different parameters are on the same order of magnitude. Spectral splatter is of similar shape whether it is due to differential gain error or differential phase error. This is no surprise, since both $g_{d}$ and $\phi_{d}$ involve $\bar{s}$ because of $v$ in (6). On the other hand, $p$ appears as a separate, constant term. The spectral splatter it generates differs in shape. Secondly, we observe that the result is strongly dependent on not only the magnitudes of the complex quantities $v_{1}$ and $v_{2}$, and $p_{1}$ and $p_{2}$, but also strongly on their relative angles. In [7], simulations are performed with $g_{d, 1}= \pm g_{d, 2}, \phi_{d, 1}= \pm \phi_{d, 2}$, and $p_{1}= \pm p_{2}$, but these scenarios are not necessarily upper or lower bounds on the spectral splatter, yet they do indicate its variance. Fig. 3 shows the dynamics of the spectral splatter as a function of the relative angle between $p_{1}$ and $p_{2}$ assuming $\left|p_{1}\right|=\left|p_{2}\right|=1.5 \%$. Plots for $\arg \left(p_{1}\right)-\arg \left(p_{2}\right)$ varying from $-\pi$ to $\pi$ with a $0.2 \pi$ increment are shown in dotted lines. The spectra can differ by over $20 \mathrm{~dB}$. The relative level of spectral splatter for a given angle depends on frequency. The curves intersect one another when $0.5 \mathrm{~W}<\left|f-f_{c}\right|<1.5 \mathrm{~W}$. However, at higher frequencies they tend to behave in a monotonic fashion. Two particular spectra are singled out in solid lines in order to illustrate this point. They are approximate upper and lower bounds on the spectral splatter for $\left|f-f_{c}\right|>1.5 \mathrm{~W}$. This simple example shows that the impact of the dc offsets varies greatly with the relative orientation of errors in the modulators, and not just their magnitude.

Finally, simulations of a realistic TWT-based LINC transmitter, that is, one afflicted with both amplifier mismatch and modulator misalignments, and a no LINC TWT transmitter are shown in Fig. 4. The no LINC transmitter is operated at levels such that the inband output power level is the same as the LINC transmitter's. The fact that two amplifiers are utilized in the LINC transmitter while only one is present in the no LINC transmitter is accounted for by shifting the output power level accordingly. In the realistic LINC transmitter the effects of both types of error appear to be additive. The LINC transmitter outperforms the no LINC transmitter by as much as $20 \mathrm{~dB}$ immediately adjacent to the message bandwidth. At frequencies where LINC may not be benefi-

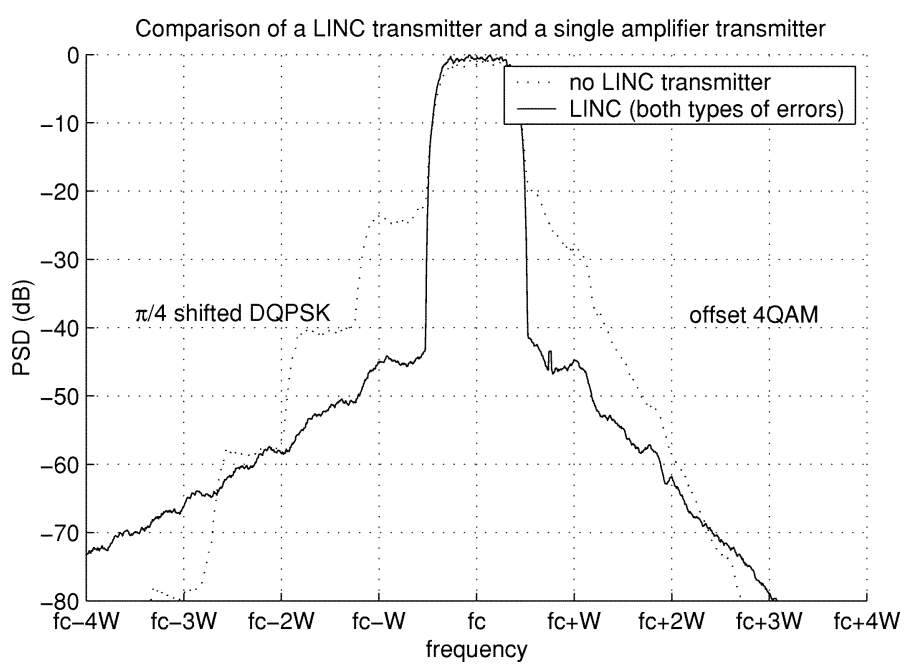

Fig. 4. Output spectra of a realistic TWT-based LINC transmitter afflicted with amplifier mismatch as well as modulator misalignments (solid line), and of a no LINC TWT transmitter. Operation is at $6 \mathrm{~dB}$ gain compression point.

cial, the spectral leakage is already below $-70 \mathrm{dBc}$ for offset 4QAM and below $-55 \mathrm{dBc}$ for $\pi / 4$ shifted DQPSK.

Further improvement of the LINC transmitter is possible through additional circuitry. For example, digital methods for compensating modulator errors can reduce the fluctuations of the magnitude of the signals envelope during upconversion in the modulators. In parallel, linearization techniques such as predistortion can be used to further reduce the impact of amplifier mismatch: Since only a small portion of the transfer curves are used, this type of technique is particularly suitable for LINC.

\section{CONCLUSION}

In this brief, we report on TWT-based LINC transmitters for high-power, high-efficiency communication applications. We demonstrate the potential performance of the TWT-based LINC transmitter assuming nonideal modulators and measured amplifier mismatch, the two main sources for distortion expected in a realistic LINC transmitter.

\section{ACKNOWLEDGMENT}

The authors would like to thank D. M. Goebel and S. E. Kubasek from Boeing EDD for providing us with data on TWT devices and for the helpful discussions.

\section{REFERENCES}

[1] R. T. Benton, U. R. Hallsten, J. A. Hill, K. P. Mallon, W. L. Menninger, and X. Zhai, "High efficiency S-band and C-band traveling wave tubes for satellite communications," in Proc. Third IEEE Int. Vac. Electron. Conf., 2002, pp. 184-185.

[2] D. C. Cox, "Linear amplification with nonlinear components," IEEE Trans. Commun., pp. 1942-1945, Dec. 1974.

[3] S. Haykin, Communication Systems, 4th ed. New York: Wiley, 2001.

[4] D. S. Komm, R. T. Benton, H. C. Limburg, W. L. Menninger, and X. Zhai, "Advances in space TWT efficiencies," IEEE Trans. Electron Devices, vol. 48, pp. 174-176, Jan. 2001.

[5] A. Lohtia, P. A. Goud, and C. G. Englefield, "An adaptive digital technique for compensating for analog quadrature modulator/demodulator impairments," in Proc. IEEE Pacific Rim Conf. Communi., Compu. Signal Process., vol. 2, 1993, pp. 447-450.

[6] R. Nagareda, K. Fukawa, and H. Suzuki, "An MMSE based calibration of LINC transmitter," in Proc. IEEE 55th Veh. Technol. Conf. , vol. 2, 2002, pp. 625-629.

[7] L. Sundström, "Spectral sensitivity of LINC transmitters to quadrature modulator misalignments," IEEE Trans. Veh. Technol., vol. 49, pp. 1474-1487, July 2000. 